\title{
Improved patient quality of life following intradural extramedullary spinal tumor resection
}

\author{
Matthew J. Viereck, BS, George M. Ghobrial, MD, Sara Beygi, MD, and James S. Harrop, MD \\ Department of Neurosurgery, Thomas Jefferson University and Jefferson Hospital for Neuroscience, Philadelphia, Pennsylvania
}

OBJECTIVE Resection significantly improves the clinical symptoms and functional outcomes of patients with intradural extramedullary tumors. However, patient quality of life following resection has not been adequately investigated. The aim in this retrospective analysis of prospectively collected quality of life outcomes is to analyze the efficacy of resection of intradural extramedullary spinal tumors in terms of quality of life markers.

METHODS A retrospective review of a single institutional neurosurgical administrative database was conducted to analyze clinical data. The Oswestry Disability Index (ODI), visual analog scale (VAS) for pain, and the EQ-5D-3 L descriptive system were used to analyze quality of life preoperatively, less than 1 month postoperatively, 1-3 months postoperatively, 3-12 months postoperatively, and more than 12 months postoperatively.

RESULTS The ODI scores increased perioperatively at the < 1-month follow-up from 36 preoperatively to 47 . Relative to preoperative values, the ODI score decreased significantly at $1-3,3-12$, and $>12$ months to 23 , 17 , and 20 , respectively. VAS scores significantly decreased from 6.1 to $3.5,2.4,2.0$, and 2.9 at the $<1$-month, 1 - to 3-, 3- to 12-, and $>12$-month follow-ups, respectively. EQ-5D mobility significantly worsened at the $<1$-month follow-up but improved at the 3 - to 12and $>12$-month follow-ups. EQ-5D self-care significantly worsened at the $<1$-month follow-up but significantly improved by the 3- to 12-month follow-up. EQ-5D usual activities improved at the 1- to 3-, 3- to 12-, and > 12-month follow-ups. EQ-5D pain and discomfort significantly improved at all follow-up points. EQ-5D anxiety and depression significantly improved at 1- to 3-month and 3- to 12-month follow-ups.

CONCLUSIONS Resection of intradural extramedullary spine tumors appears to significantly improve patient quality of life by decreasing patient disability and pain and by improving each of the EQ-5D domains.

http://thejns.org/doi/abs/10.3171/2016.4.SPINE151149

KEY WORDS spinal tumor; intradural extramedullary; schwannoma; nerve sheath tumor; neurofibroma; neurofibromatosis; intradural tumor; quality of life; functional outcome; oncology

I NTRADURAL extramedullary spinal tumors are rare neoplasms that can cause significant pain, gait disturbances, sensory changes, and decreased longevity. While the majority of spinal tumor pathologies in the intradural extramedullary compartments of the spine are schwannomas, meningiomas, and neurofibromas, these histologically benign tumors can cause profound impairment on quality of life through pain and myeloradicular symptoms caused by direct compressive effects. Management of patients experiencing severe symptoms frequently involves tumor resection. The impact of operative resection of intradural extramedullary spinal tumors on patient quality of life has not been previously established in the literature. Furthermore, the time course of patient improvement has yet to be described. We present a large series of patients with intradural extramedullary tumors with an emphasis on postoperative quality of life outcomes.

\section{Methods}

A retrospective study of prospectively collected data from a neurosurgical administrative electronic database was conducted from 2004 to 2014. Prior university institutional review board approval had been obtained.

\section{Inclusion Criteria}

A Current Procedural Terminology code search (63265, $63267,63275,63276,63277,63280,63281)$ was conducted to identify patients with spinal tumors. Patients were selected based on the following criteria: presence of an 
intradural extramedullary tumor of the spine; pathology positive for schwannoma, neurofibroma, ependymoma, or meningioma; the patient underwent resection; and the patient had completed at a minimum a preoperative and a postoperative quality of life assessment.

\section{Exclusion Criteria}

Patients who did not have both a preoperative and a postoperative follow-up data point were excluded. Patients with extradural and intramedullary tumors of the spine, tumors WHO Grade II or greater, and patients who had undergone prior spinal tumor resection were excluded.

\section{Data Collection}

Patient information and tumor data were collected using inpatient and outpatient electronic medical records.

\section{Functional Outcomes Measures}

Health status was evaluated using the patient-reported Oswestry Disability Index 2.0 (ODI), the EQ-5D-3 L descriptive system (EQ-5D), and visual analog scale (VAS) for pain completed during preoperative and postoperative outpatient visits. ODI items that were unanswered were interpreted as not significantly affecting patient disability and were scored as 0 points. ODI forms that included 3 or more unanswered items were not included in analysis. VAS for pain was assessed using a $10-\mathrm{cm}$ line in which patients marked their level of pain from 0 (no pain) to 10 (worst pain). Values were converted to the nearest integer. Each EQ-5D-3 L domain (mobility, self-care, performing usual activities, pain and discomfort, and anxiety and depression) was assessed through patient-completed reports of having no problems, some problems, or extreme problems for each domain.

\section{Statistical Analysis}

Statistical analysis was conducted using IBM SPSS Statistics (version 22.0, IBM). Mann-Whitney U-tests were used to determine the influence of tumor resection on ODI and VAS scores. The influence of tumor resection on each EQ-5D domain was assessed using Fisher's exact test. Significance was defined as $p<0.05$.

\section{Results}

\section{Baseline Characteristics}

Forty-four patients were identified who met our inclusion criteria (intradural extramedullary tumor resection, preoperative and postoperative ODI, EQ-5D, and/or VAS for pain scores). Twenty-eight patients were female $(63.6 \%)$ and 16 were male $(36.4 \%)$. The mean age at the time of operation was $53.2 \pm 13.6$ years $( \pm \mathrm{SD})$.

\section{Tumor Characteristics}

The most frequent tumor pathology was schwannoma $(\mathrm{n}=26,59.1 \%)$, followed by meningioma $(\mathrm{n}=14,31.8 \%)$, ependymoma $(\mathrm{n}=3,6.8 \%)$, and neurofibroma $(\mathrm{n}=1$, $2.3 \%)$. Nine tumors $(20.5 \%)$ were located in the cervical region, $12(27.3 \%)$ were located in the thoracic region, 2 $(4.5 \%)$ at the thoracolumbar junction, 18 (40.9\%) in the lumbar region, $1(2.3 \%)$ in the lumbosacral region, and 2 $(4.5 \%)$ in the sacral region. The mean number of vertebral levels occupied by tumors was 1.84 (Table 1).

\section{Surgical Characteristics}

Forty-three patients (97.7\%) underwent a laminectomy followed by tumor resection. Seven patients (15.9\%) underwent an instrumented fusion while 2 patients (4.5\%) underwent anterior interbody instrumented fusion. Fortytwo patients $(95.5 \%)$ had complete tumor resection. The mean length of stay was 5.8 days (Table 1 ).

\section{Patient Follow-Up}

Twenty-nine patients $(65.9 \%)$ had a follow-up visit and completed the ODI, EQ-5D, and/or VAS for pain within 1 month following surgery, 31 patients $(70.5 \%)$ between 1 and 3 months following surgery, 32 (65.9\%) between 3 and 12 months following surgery, and 20 patients (45.5\%) greater than 12 months following surgery (Table 2; Fig. 1).

\section{Oswestry Disability Index}

The mean preoperative ODI score was 36 . At the less

TABLE 1. Procedure and surgical characteristics*

\begin{tabular}{|c|c|}
\hline Variable & Value \\
\hline \multicolumn{2}{|l|}{ Lesion type (\%) } \\
\hline Schwannoma & $26(59.1)$ \\
\hline Meningioma & $14(31.8)$ \\
\hline Ependymoma & $3(6.8)$ \\
\hline Neurofibroma & $1(2.3)$ \\
\hline \multicolumn{2}{|l|}{ Location } \\
\hline Cervical & $9(20.5)$ \\
\hline Thoracic & $12(27.3)$ \\
\hline Thoracolumbar & $2(4.5)$ \\
\hline Lumbar & $18(40.9)$ \\
\hline Lumbosacral & $1(2.3)$ \\
\hline Sacral & $2(4.5)$ \\
\hline Mean no. of tumor levels & $1.84 \pm 0.914$ \\
\hline \multicolumn{2}{|l|}{ No. of procedures } \\
\hline Laminectomy & $43(97.7)$ \\
\hline Posterior instrumentation & $7(15.9)$ \\
\hline Anterior interbody instrumented fusion & $2(4.5)$ \\
\hline Mean length of stay in days & $5.8 \pm 3.0$ \\
\hline Complete resection & $42(95.5)$ \\
\hline \multicolumn{2}{|l|}{ Complications } \\
\hline Pseudomeningocele & $4(9.1)$ \\
\hline Wound infection & $2(4.5)$ \\
\hline New neurological symptoms & $2(4.5)$ \\
\hline Reoperation & $2(4.5)$ \\
\hline Deep vein thrombosis & $1(2.3)$ \\
\hline Incontinence & $1(2.3)$ \\
\hline Pneumonia & $1(2.3)$ \\
\hline
\end{tabular}

* Values are presented as the number of lesions (\%) unless noted otherwise. Mean values are presented as the mean \pm SD. 
TABLE 2. Patient follow-up

\begin{tabular}{lc}
\hline Time Point & No. of Patients \\
\hline Preop & $44(100)$ \\
\hline$<1$ mo & $29(65.9)$ \\
\hline $1-3$ mos & $31(70.5)$ \\
\hline $3-12$ mos & $32(65.9)$ \\
\hline$>12$ mos & $20(45.5)$ \\
\hline
\end{tabular}

than 1-month follow-up, the mean ODI significantly increased from the preoperative value to $47(\mathrm{p}=0.033)$. At the 1- to 3-month follow-up, the mean ODI decreased significantly from the preoperative value to $23(\mathrm{p}=0.016)$. Mean ODI scores were significantly less at the 3- to 12-month follow-up (Score 17, p < 0.001) and the more than 12-month follow-ups (Score 20, $p=0.010$ ) compared with the preoperative value (Score 36) (Table 3).

\section{VAS for Pain}

The mean preoperative VAS for pain value was 6.1. At the less than 1-month follow-up, the mean VAS for pain significantly decreased from the preoperative value to 3.5 $(\mathrm{p}=0.002)$. The VAS for pain value was also significantly lower than the preoperative value at the 1 - to 3 -month (2.4, $\mathrm{p}<0.001), 3$ - to 12 -month $(2.0, \mathrm{p}<0.001)$, and more than 12-month follow-up visits $(2.9, \mathrm{p}=0.011)$ (Table 3$)$.

\section{EQ-5D Mobility}

Preoperatively, 13 patients (29.5\%) reported no problems with mobility, 30 patients $(68.2 \%)$ reported some problems with mobility, and 1 patient (2.3\%) reported extreme problems with mobility. At the less than 1-month follow-up, 2 patients (7.4\%) reported no problems with mobility, 25 patients $(92.6 \%)$ reported some problems with mobility, and no patient reported extreme problems with mobility (significantly worsened mobility compared with preoperative values, $\mathrm{p}=0.036$ ). At the 1 - to 3 -month follow-up, 10 patients $(32.3 \%)$ reported no problems with mobility, 21 patients $(67.7 \%)$ reported some problems with mobility, and no patient reported extreme problems with mobility (no significant difference compared with preoperative values, $\mathrm{p}=1.000$ ). At the 3 - to 12 -month follow-up, 18 patients (62.1\%) reported no problems with mobility, 11 patients $(37.9 \%)$ reported some problems with mobility, and no patient reported extreme problems with mobility (significantly improved compared with preoperative values, $\mathrm{p}=0.011$ ). At the more than 12-month follow-up, 12 patients (63.2\%) reported no problems with mobility, 6 patients reported some problems with mobility (31.6\%), and 1 patient (5.3\%) reported extreme problems with mobility (significantly improved compared with preoperative values, $\mathrm{p}=0.014$ ) $($ Table 4$)$.

\section{EQ-5D Self-Care}

Preoperatively, 29 patients (65.9\%) reported no problems with self-care, 15 (34.1\%) reported some problems with self-care, and no patient reported extreme problems with self-care. At the less than 1-month follow-up, 9 pa-

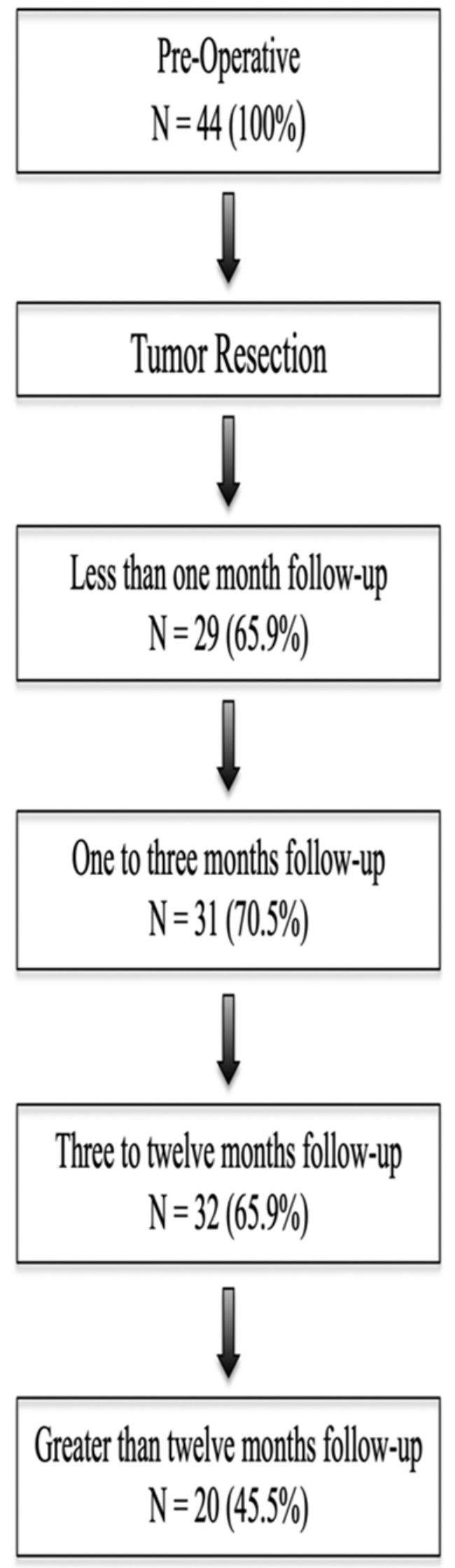

FIG. 1. Chart showing the number of patients at each follow-up. 
TABLE 3. Preoperative and postoperative ODI and VAS scores*

\begin{tabular}{lcrcr}
\hline Time Point & ODI & p Value $\dagger$ & VAS & p Value $\dagger$ \\
\hline Preop & $36 \pm 23$ & & $6.1 \pm 3.1$ & \\
\hline$<1$ mo & $47 \pm 17$ & 0.033 & $3.5 \pm 2.3$ & 0.002 \\
\hline $1-3$ mos & $23 \pm 20$ & 0.016 & $2.4 \pm 2.5$ & $<0.001$ \\
\hline $3-12$ mos & $17 \pm 20$ & $<0.001$ & $2.0 \pm 2.6$ & $<0.001$ \\
\hline$>12$ mos & $20 \pm 27$ & 0.010 & $2.9 \pm 3.7$ & 0.011 \\
\hline
\end{tabular}

* Scores are presented as the mean \pm SD.

$\dagger$ Compared with preoperatively.

tients $(33.3 \%)$ reported no problems with self-care, 18 patients $(66.7 \%)$ reported some problems with care, and no patient reported extreme problems with self-care (significantly worsened self-care compared with preoperative values, $p=0.014)$. At the 1- to 3-month follow-up, 20 patients $(64.5 \%)$ reported no problems with self-care, 11 patients $(35.5 \%)$ reported some problems with self-care, and no patient reported extreme problems with self-care (no significant difference compared with preoperative values, $\mathrm{p}=1.000)$. At the 3 - to 12 -month follow-up, 26 patients $(89.7 \%)$ reported no problems with self-care, 3 patients $(10.3 \%)$ reported some problems with self-care, and no patient reported extreme problems with self-care (significantly improved compared with preoperative values, $\mathrm{p}=0.027)$. At the greater than 12-month follow-up, 15 patients $(78.9 \%)$ reported no problems with self-care, 4 patients (21.1\%) reported some problems with self-care, and no patient reported extreme problems with self-care (no significant difference compared with preoperative values, $\mathrm{p}=0.379$ ) (Table 4).

\section{EQ-5D Usual Activities}

Preoperatively, 5 patients (11.4\%) reported no problems performing usual activities, 28 patients $(63.6 \%)$ reported some problems performing usual activities, and 11 patients $(25.0 \%)$ reported extreme problems performing usual activities. At the less than 1-month follow-up, 1 patient $(3.7 \%)$ reported no problems performing usual activities, 15 patients $(55.6 \%)$ reported some problems performing usual activities, and 11 patients $(40.7 \%)$ reported extreme problems performing usual activities (no significant difference compared with preoperative values, $\mathrm{p}=$ $0.286)$. At the 1 - to 3 -month follow-up, 8 patients $(25.8 \%)$ reported no problems performing usual activities, 22 patients $(71.0 \%)$ reported some problems performing usual activities, and 1 patient (3.2\%) reported extreme problems performing usual activities (significantly improved compared with preoperative values, $\mathrm{p}=0.018)$. At the 3 to 12 -month follow-up, 14 patients $(48.3 \%)$ reported no problems performing usual activities, 13 patients $(44.8 \%)$ reported some problems performing usual activities, and 2 patients (6.9\%) reported extreme problems performing usual activities (significantly improved compared with preoperative values, $\mathrm{p}=0.001)$. At the longer than 12-month follow-up, 9 patients $(47.4 \%)$ reported no problems performing usual activities, 8 patients (42.1\%) reported some problems performing usual activities, and 2 patients $(10.5 \%)$ reported extreme problems performing usual activities (significantly improved compared with preoperative values, $\mathrm{p}=0.009$ ) (Table 4$)$.

\section{EQ-5D Pain and Discomfort}

Preoperatively, 3 patients $(7.0 \%)$ reported no problems with pain and discomfort, 26 patients $(60.5 \%)$ reported some problems with pain and discomfort, and 14 (32.6\%) reported extreme problems with pain and discomfort. At the less than 1-month follow-up, 2 patients (7.4\%) reported no problems with pain and discomfort, 23 (85.2\%) reported some problems with pain and discomfort, and 2 patients $(7.4 \%)$ reported extreme problems with pain and discomfort (significantly improved compared with preoperative values, $p=0.036$ ). At the 1 - to 3 -month followup, 9 patients $(29.0 \%)$ reported no problems with pain and discomfort, 20 patients $(64.5 \%)$ reported some problems with pain and discomfort, and 2 patients $(6.5 \%)$ reported

TABLE 4. Preoperative and postoperative EQ-5D descriptive system scores*

\begin{tabular}{|c|c|c|c|c|}
\hline \multirow[b]{2}{*}{ Domain \& Time Point } & \multicolumn{3}{|c|}{ No. of Patients (\%) } & \multirow[b]{2}{*}{$p$ Value $†$} \\
\hline & None & Some & Severe & \\
\hline \multicolumn{5}{|l|}{ Mobility } \\
\hline Preop & $13(29.5)$ & $30(68.2)$ & $1(2.3)$ & \\
\hline$<1 \mathrm{mo}$ & $2(7.4)$ & $25(92.6)$ & $0(0.0)$ & 0.036 \\
\hline $1-3$ mos & $10(32.3)$ & $21(67.7)$ & $0(0.0)$ & 1.000 \\
\hline $3-12$ mos & $18(62.1)$ & $11(37.9)$ & $0(0.0)$ & 0.011 \\
\hline$>12$ mos & $12(63.2)$ & $6(31.6)$ & $1(5.3)$ & 0.014 \\
\hline \multicolumn{5}{|l|}{ Self-care } \\
\hline Preop & $29(65.9)$ & $15(34.1)$ & $0(0.0)$ & \\
\hline$<1 \mathrm{mo}$ & $9(33.3)$ & $18(66.7)$ & $0(0.0)$ & 0.014 \\
\hline $1-3 \mathrm{mos}$ & $20(64.5)$ & $11(35.5)$ & $0(0.0)$ & 1.000 \\
\hline 3-12 mos & $26(89.7)$ & $3(10.3)$ & $0(0.0)$ & 0.027 \\
\hline$>12$ mos & $15(78.9)$ & $4(21.1)$ & $0(0.0)$ & 0.379 \\
\hline \multicolumn{5}{|l|}{ Usual activities } \\
\hline Preop & $5(11.4)$ & $28(63.6)$ & $11(25.0)$ & \\
\hline$<1 \mathrm{mo}$ & $1(3.7)$ & $15(55.6)$ & $11(40.7)$ & 0.286 \\
\hline $1-3$ mos & $8(25.8)$ & $22(71.0)$ & $1(3.2)$ & 0.018 \\
\hline $3-12$ mos & $14(48.3)$ & $13(44.8)$ & $2(6.9)$ & 0.001 \\
\hline$>12$ mos & $9(47.4)$ & $8(42.1)$ & $2(10.5)$ & 0.009 \\
\hline \multicolumn{5}{|l|}{ Pain \& discomfort } \\
\hline Preop & $3(7.0)$ & $26(60.5)$ & $14(32.6)$ & \\
\hline$<1 \mathrm{mo}$ & $2(7.4)$ & $23(85.2)$ & $2(7.4)$ & 0.036 \\
\hline $1-3 \mathrm{mos}$ & $9(29.0)$ & $20(64.5)$ & $2(6.5)$ & 0.004 \\
\hline $3-12$ mos & $12(41.4)$ & $15(51.7)$ & $2(6.9)$ & 0.001 \\
\hline$>12$ mos & $8(44.4)$ & $6(33.3)$ & $4(22.2)$ & 0.004 \\
\hline \multicolumn{5}{|l|}{ Anxiety \& depression } \\
\hline Preop & $15(35.7)$ & $21(50.0)$ & $6(14.3)$ & \\
\hline$<1 \mathrm{mo}$ & $17(63.0)$ & $9(33.3)$ & $1(3.7)$ & 0.078 \\
\hline $1-3$ mos & $21(67.7)$ & $8(25.8)$ & $2(6.5)$ & 0.034 \\
\hline $3-12$ mos & $21(72.4)$ & $5(17.2)$ & $3(10.3)$ & 0.007 \\
\hline$>12$ mos & $11(61.1)$ & $6(33.3)$ & $1(5.6)$ & 0.217 \\
\hline
\end{tabular}


extreme problems with pain and discomfort (significantly improved compared with preoperative values, $\mathrm{p}=0.004$ ). At the 3- to 12-month follow-up, 12 patients (41.4\%) reported no problems with pain and discomfort, 15 patients $(51.7 \%)$ reported some problems with pain and discomfort, and 2 patients $(6.9 \%)$ reported extreme problems with pain and discomfort (significantly improved compared with preoperative values, $\mathrm{p}=0.001)$. At the longer than 12-month follow-up, 8 patients (44.4\%) reported no problems with pain and discomfort, 6 patients (33.3\%) reported some problems with pain and discomfort, and 4 patients (22.2\%) reported extreme problems with pain and discomfort (significantly improved compared with preoperative values, $\mathrm{p}=0.004)$ (Table 4$)$.

\section{EQ-5D Anxiety and Depression}

Preoperatively, 15 patients $(35.7 \%)$ reported no problems with anxiety and depression, 21 patients (50.0\%) reported some problems with anxiety and depression, and 6 patients (14.3\%) reported extreme problems with anxiety and depression. At the less than 1-month follow-up, 17 patients $(63.0 \%)$ reported no problems with anxiety and depression, 9 patients (33.3\%) reported some problems with anxiety and depression, and 1 patient (3.7\%) reported extreme problems with anxiety and depression (no significant difference compared with preoperative values, $\mathrm{p}=$ 0.078). At the 1- to 3 -month follow-up, 21 patients $(67.7 \%)$ reported no problems with anxiety and depression, 8 patients $(25.8 \%)$ reported some problems with anxiety and depression, and 2 patients (6.5\%) reported extreme problems with anxiety and depression (significantly improved compared with preoperative values, $\mathrm{p}=0.034)$. At the 3 to 12-month follow-up, 21 patients $(72.4 \%)$ reported no problems with anxiety and depression, 5 patients (17.2\%) reported some problems with anxiety and depression, and 3 patients $(10.3 \%)$ reported extreme problems with anxiety and depression (significantly improved compared with preoperative values, $\mathrm{p}=0.007)$. At the greater than 12-month follow-up, 11 patients $(61.1 \%)$ reported no problems with anxiety and depression, 6 patients (33.3\%) reported some problems with anxiety and depression, and 1 patient (5.6\%) reported extreme problems with anxiety and depression (no significant difference compared with preoperative values, $\mathrm{p}=0.217$ ) (Table 4 ).

\section{Complications}

Four patients $(9.1 \%)$ developed a pseudomeningocele following surgery. Two patients $(4.5 \%)$ had a surgical site infection requiring irrigation and debridement. Two patients $(4.5 \%)$ developed new neurological symptoms of upper-extremity weakness following surgery. One patient $(2.0 \%)$ each experienced deep vein thrombosis, incontinence, and pneumonia. Two patients $(4.5 \%)$ required reoperation; one for debridement of a posterior wound seroma and one for removal of a keloid (Table 1).

\section{Discussion}

The effectiveness of tumor resection for treatment of the clinical symptoms of intradural extramedullary tumors has been well documented. ${ }^{3,46-8}$ Studies have also shown improved functional outcome following tumor resection. ${ }^{1,7}$ However, there is minimal evidence demonstrating improvement of patient quality of life compared with preoperative values. One large series by Tarantino and colleagues looked at EQ-5D visual analog scores postoperatively and showed that patients reported a mean score of $61.78 \%$, interpreted as a middle-high quality of life. 9 However, no preoperative values were measured and the change in quality of life could not be assessed.

\section{Oswestry Disability Index}

In the present study, we used patient-reported ODI, VAS, and EQ-5D scores to evaluate patient disability, pain, and health status, respectively. Preoperatively, patients reported significant disability with a mean ODI score of 36 . Following tumor resection, the mean ODI score initially increased as demonstrated by a mean ODI score of 47 at the less than 1-month follow-up. However, this discrepancy was likely perioperative pain as the symptoms dramatically improved thereafter. For the 1- to 3-month, 3- to 12-month, and greater than 12-month follow-ups, patient disability was significantly improved compared with baseline. Clinically significant changes in ODI scores as determined by the minimum clinically important difference was reported as 12.8 points for ODI by Copay et al. ${ }^{2}$ For the current study, the changes in ODI scores were $+11,-13$, -19 , and -16 comparing the preoperative value to the less than 1-month, 1- to 3-month, 3- to 12-month, and greater than 12-month follow-ups, respectively. These results suggest that at the less than 1-month follow-up, the increase in ODI is not clinically significant whereas the decrease in ODI scores at the 1- to 3-, 3- to 12-, and greater than 12-month follow-ups is clinically significant.

\section{VAS Pain}

Patients reported substantial preoperative pain, due to both axial and radiculopathic symptoms. Following tumor resection, patients reported significant improvement in pain at each follow-up visit. In contrast to disability scores, pain improved at the less than 1-month follow-up, suggesting that tumor resection improves patient pain earlier than patient disability. Similar studies have also demonstrated the effectiveness of intradural extramedullary tumor resection for relief of pain. ${ }^{1,5}$ To our knowledge, the present study is the first to demonstrate significant pain improvement in the early postoperative period.

\section{EQ-5D}

A change in the relative frequencies favoring patients reporting some or extreme problems preoperatively to none or some problems postoperatively was interpreted as improvement in the respective EQ-5D domain. Preoperatively, a large percentage of patients reported some or extreme problems with mobility (70.5\%), performing usual activities (88.6\%), pain and discomfort (93.0\%), and anxiety and depression (64.3\%). Only $34.1 \%$ of patients reported problems with self-care preoperatively. Improvement was observed in all 5 domains. Improvement in mobility was observed at the 3- to 12-month and greater than 12-month follow-ups. Improvement in self-care was observed at the 
3- to 12-month follow-up. Improvement in performing usual activities was observed at the 1- to 3-month, 3- to 12-month, and greater than 12-month follow-ups. The pain and discomfort domain score was improved at all followups. The anxiety and depression domain score was improved at the 1- to 3-month and 3- to 12-month follow-ups. Two domains (mobility and self-care) had a significant increase in the proportion of patients reporting some or severe problems postoperatively. Three domains (mobility, usual activities, and pain and discomfort) improved postoperatively and stayed improved from baseline at each consecutive follow-up. Two domains (self-care and anxiety and depression) improved significantly but returned to near baseline levels, lacking statistically significant improvement on consecutive follow-ups. The reason for this is multifactorial and likely related to a smaller sample size at the greater than 12-month follow-up.

There are limitations of this study that are inherent with all retrospective studies. The findings of improved quality of life from resection of intradural extramedullary tumors must be reconciled with the significant drop-off in patient follow-up between 3, 12, and greater than 12 months (Fig. 1). Furthermore, a long-held belief that patients with satisfactory outcomes are more likely to self-report must be a consideration when drawing these conclusions.

We present here the first evidence of improved quality of life in patients following intradural extramedullary tumor resection. We further describe patient improvement by detailing the time course of patient improvement.

\section{Conclusions}

Overall, the data demonstrate that tumor resection significantly improved disability, pain, and health status in patients with intradural extramedullary spinal tumors, suggesting improvement in overall quality of life.

\section{References}

1. Ahn DK, Park HS, Choi DJ, Kim KS, Kim TW, Park SY: The surgical treatment for spinal intradural extramedullary tumors. Clin Orthop Surg 1:165-172, 2009

2. Copay AG, Glassman SD, Subach BR, Berven S, Schuler TC, Carreon LY: Minimum clinically important difference in lumbar spine surgery patients: a choice of methods using the Oswestry Disability Index, Medical Outcomes Study ques- tionnaire Short Form 36, and pain scales. Spine J 8:968-974, 2008

3. Jenkinson MD, Simpson C, Nicholas RS, Miles J, Findlay GF, Pigott TJ: Outcome predictors and complications in the management of intradural spinal tumours. Eur Spine J 15:203-210, 2006

4. Mehta AI, Adogwa O, Karikari IO, Thompson P, Verla T, Null UT, et al: Anatomical location dictating major surgical complications for intradural extramedullary spinal tumors: a 10-year single-institutional experience. J Neurosurg Spine 19:701-707, 2013

5. Nzokou A, Weil AG, Shedid D: Minimally invasive removal of thoracic and lumbar spinal tumors using a nonexpandable tubular retractor. J Neurosurg Spine 19:708-715, 2013

6. Parsa AT, Lee J, Parney IF, Weinstein P, McCormick PC, Ames C: Spinal cord and intradural-extraparenchymal spinal tumors: current best care practices and strategies. J Neurooncol 69:291-318, 2004

7. Setzer M, Vatter H, Marquardt G, Seifert V, Vrionis FD: Management of spinal meningiomas: surgical results and a review of the literature. Neurosurg Focus 23(4):E14, 2007

8. Song KW, Shin SI, Lee JY, Kim GL, Hyun YS, Park DY: Surgical results of intradural extramedullary tumors. Clin Orthop Surg 1:74-80, 2009

9. Tarantino R, Donnarumma P, Nigro L, Rullo M, Santoro A, Delfini R: Surgery of intradural extramedullary tumors: retrospective analysis of 107 cases. Neurosurgery 75:509-514, 2014

\section{Disclosures}

The authors report no conflict of interest concerning the materials or methods used in this study or the findings specified in this paper.

\section{Author Contributions}

Conception and design: Harrop. Acquisition of data: Viereck, Beygi. Analysis and interpretation of data: Ghobrial, Viereck, Beygi. Drafting the article: Ghobrial, Viereck, Beygi. Critically revising the article: Harrop, Ghobrial. Reviewed submitted version of manuscript: Harrop, Ghobrial. Study supervision: Harrop.

\section{Correspondence}

James S. Harrop, Department of Neurosurgery, Thomas Jefferson University and Jefferson Hospital for Neuroscience, 909 Walnut St., 2nd Fl., Philadelphia, PA 19107.email: james.harrop@ jefferson.edu. 